\title{
Primary Retroperitoneal Mucinous Cystadenoma
}

\author{
Seok Youn Lee, Weon Cheol Han ${ }^{1}$ \\ Departments of Surgery and ${ }^{1}$ Pathology, Wonkwang University Sanbon Hospital, Wonkwang University School of Medicine, Iksan, Korea
}

\begin{abstract}
Mucinous cystadenomas and cystadenocarcinomas of the ovary are clinically and histopathologically well-established common tumors. However, primary retroperitoneal mucinous cystic tumors are extremely rare, and although their histopathogenesis is still uncertain, several theories have been proposed. Most authors suggest that they develop through mucinous metaplasia in a preexisting mesothelium-lined cyst. An accurate preoperative diagnosis of these tumors is difficult because no effective diagnostic measures have been established. Delay in diagnosis and treatment of this tumor may be fatal for the patient because of complications such as rupture, infection, and malignant transformation. We describe the case of a 31-year-old woman with abdominal pain and a palpable mass. Computed tomography of the abdomen revealed a retroperitoneal cystic mass, which was resected successfully through laparoscopy. Histopathological examination of the resected mass confirmed the diagnosis of a primary retroperitoneal mucinous cystadenoma. The patient was discharged on postoperative day 5 without any complications.
\end{abstract}

Keywords: Retroperitoneal neoplasms; Mucinous cystadenoma

\section{INTRODUCTION}

Primary retroperitoneal mucinous cystadenomas (PRMCs) are very rare, even though mucinous cystadenomas are common ovarian tumors [1]. The retroperitoneal location of cystic tumors resembling ovarian mucinous cystadenoma is extremely rare. PRMCs are observed more frequently in women, with only 4 cases having been found in men [2]. Most patients are diagnosed without specific symptoms, but, like most retroperitoneal masses, they cause symptoms when growing large enough to exert pressure or have an obstructive effect on adjacent organs [3]. The diagnosis of retroperitoneal tumors is important, but difficult, as serological investigations, ultrasonography, computed tomography (CT), and magnetic resonance imaging (MRI), although useful, cannot allow a confident diagnosis. Once diagnosed, the tumor should be completely removed because of the risk of infection, re-

Received: September 22, 2015 - Accepted: October 21, 2015

Correspondence to: Seok Youn Lee, M.D.

Department of Surgery, Wonkwang University Sanbon Hospital, Wonkwang

University School of Medicine, 327 Sanbon-ro, Gunpo 15865, Korea

Tel: +82-31-390-2218, Fax: +82-31-390-2983

E-mail: sylee314@hotmail.com

(C) 2016 The Korean Society of Coloproctology

This is an open-access article distributed under the terms of the Creative Commons Attribution NonCommercial License (http://creativecommons.org/licenses/by-nc/4.0) which permits unrestricted noncommercial use, distribution, and reproduction in any medium, provided the original work is properly cited. currence and malignant degeneration, even though the tumor behaves in a benign fashion with no recurrences.

PRMCs are rare tumors, as indicated by the small number of cases reported in the literature. Approximately 50 cases of primary retroperitoneal mucinous cystadenocarcinomas have been reported in the international literature, but only 29 cases of a PRMC have been described [2]. This is 30th case in the literature, and the 2 nd case reported in Korea [4]. We report herein the case of a 31-year-old woman in whom laparoscopic resection of a PRMC was successfully performed.

\section{CASE REPORT}

A 31-year-old woman presented to our institution with a 6-month history of intermittent abdominal pain and a palpable mass in the left lower abdomen. She had no past medico-surgical history. Physical examination revealed a painless, palpable mass in the left lower quadrant of the abdomen. In cardiopulmonary auscultation, nothing unusual was detected. At the time of admission, her blood pressure, temperature, and pulse were $120 / 70 \mathrm{mmHg}$, $36.3^{\circ} \mathrm{C}$, and 70 beats per minute, respectively. The laboratory data were within reference ranges. Tumor markers, including carbohydrate antigen 19-9 (CA 19-9), CA 125, alpha-fetoprotein ( $\alpha-\mathrm{FP})$, and carcinoembryonic antigen (CEA), were normal. The abdomen film demonstrated a large hazy mass located over the left lower side of the abdomen and displacing the left-side colon me- 


\section{Coloproctology \\ Primary Retroperitoneal Mucinous Cystadenoma \\ Seok Youn Lee and Weon Cheol Han}

dially (Fig. 1A). CT of the abdomen confirmed a unilocular cystic mass with multiple peripheral wall calcifications, measuring 8.9 $\mathrm{cm} \times 9.7 \mathrm{~cm} \times 10 \mathrm{~cm}$ in size and located in the retroperitoneum in the left lower quadrant of the abdomen (Fig. 1B, C). It was separated from the left kidney and ovary. Ascites were not detected. Provisional diagnosis included retroperitoneal or colonic lymphangioma and duplication of the colon. Because a retroperitoneal cystic mass was considered, the patient underwent surgical exci- sion of the tumor. The procedure was initiated and completed via the laparoscopic approach.

On laparoscopy, the cyst was found to be located behind the posterior peritoneum of the descending colon. The white line of Toldt was divided to expose the cystic mass. It was tightly adhered to the left colon and its posterior peritoneum, but it had not invaded any adjacent organs (Fig. 2A). The uterus and the ovary were normal. The retroperitoneal tumor was completely removed,
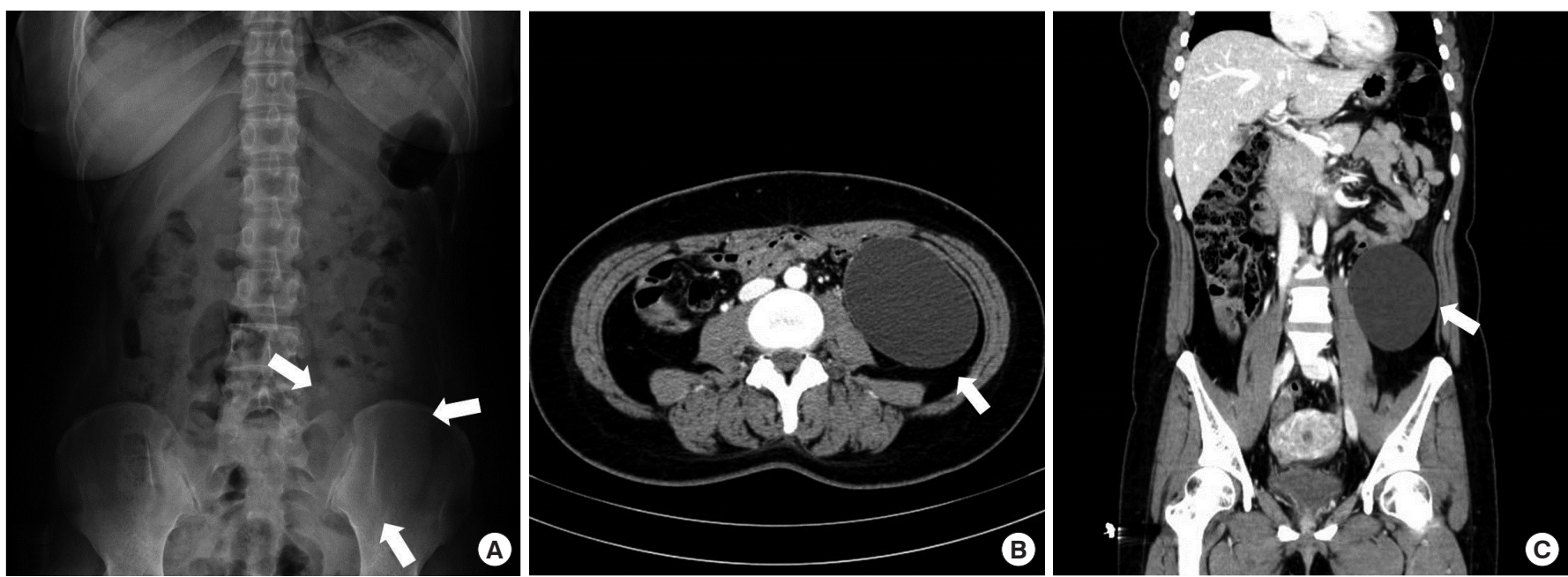

Fig. 1. Abdominal radiograph showing a hazy mass located over the left lower side of the abdomen displacing the left-side colon medially (arrows). (A) Abdominopelvic computed tomography scan showing a well-defined homogenous hypodense mass, measuring $8.9 \mathrm{~cm} \times 9.7 \mathrm{~cm} \times$ $10 \mathrm{~cm}$ in size, occupying the left retroperitoneal space and abutting the descending colon with medial displacement (arrows). Axial view (B) and coronal view $(\mathrm{C})$.
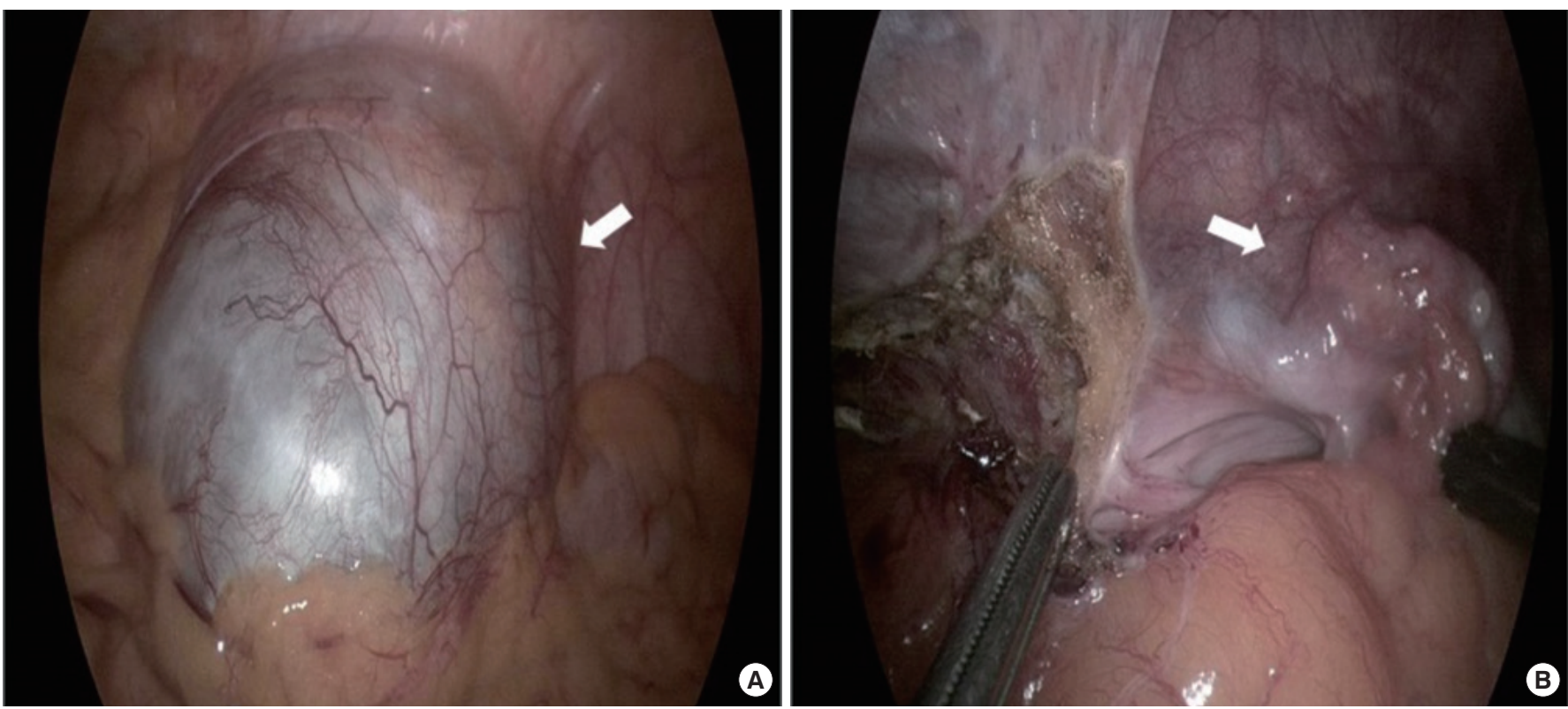

Fig. 2. Intraoperative findings by laparoscopy. (A) Unilocular cystic mass in the retroperitoneum (arrow). (B) No pedicle linking the mass to the digestive or gynecologic structure was detected. The left ovary was present and normal (arrow). 

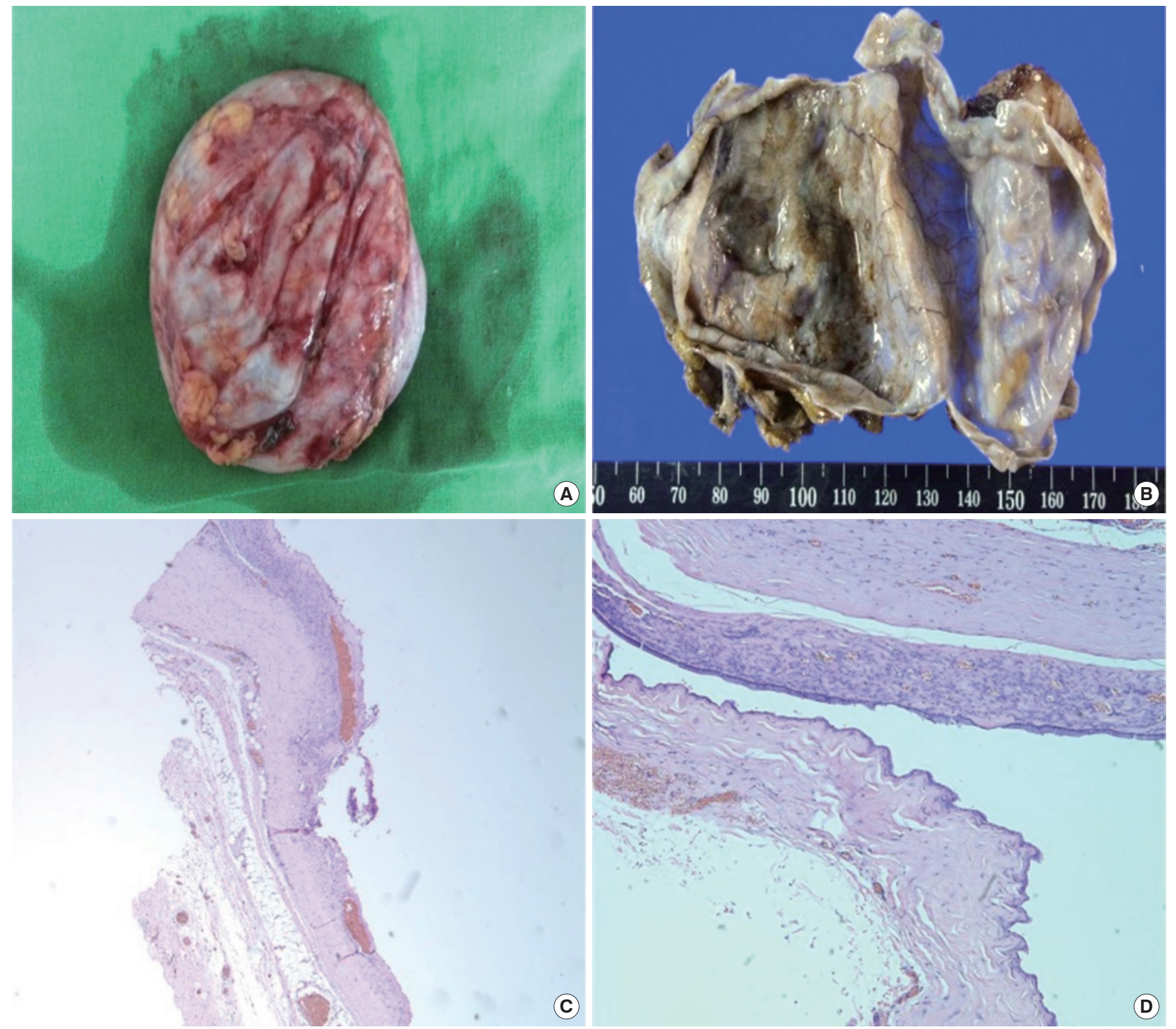

Fig. 3. Gross findings of the resected specimen. (A) The cystic mass measured $10 \mathrm{~cm} \times 9 \mathrm{~cm} \times 9 \mathrm{~cm}$ in size, and the external surface showed no connection to any viscera. (B) On the cut section, the cystic mass was composed of a unilocular cyst and clear mucinous fluid. (C) The stroma consisted of fibrocollagenous tissue with no ovarian components (H\&E, $\times 40)$. (D) The cyst wall was lined with a single layer of mucinous epithelial cells $(\mathrm{H} \& \mathrm{E}, \times 200)$.

without spillage of its contents, through the laparoscope, and a combined resection of the associated organs was not performed (Fig. 2B). No complications were observed during the postoperative course.

A huge retroperitoneal cystic mass, measuring $8 \mathrm{~cm} \times 9 \mathrm{~cm} \times 9$ $\mathrm{cm}$ in external diameter, having finely vascularized walls and containing clear mucinous fluid, was resected (Fig. 3A, B). Histopathological assessment of the resected mass disclosed a picture of a mucinous cystadenoma lined by a single layer of columnar epithelium with mucin production and surrounded by dense fibrous tissues (Fig. 3C, D). A diagnosis of a PRMC was made. The postoperative course was uneventful, and the patient was discharged 5 days after surgery without any complications.

\section{DISCUSSION}

Retroperitoneal tumors account for less than $0.2 \%$ of all neoplasms. Primary retroperitoneal mucinous tumors are rare, and 
although they share a histological similarity to ovarian mucinous cystadenomas, they can arise at any location in the retroperitoneum without attachment to the ovary [5]. The first description of a PRMC was undertaken by Handfield-Jones [6] in 1924 in his study on retroperitoneal cysts. As there are no epithelial cells in the retroperitoneum, the occurrence of this tumor type is extremely rare. To the best of our knowledge, this is the 30th report of a PRMC in the literature [2].

Mucinous cystadenomas are more commonly found in the pancreas and the ovary; therefore, the origin of mucinous cystadenomas in the retroperitoneum is widely debated. Two main theories may explain the histogenesis of a retroperitoneal mucinous cystadenoma. One theory involves the seeding of ectopic ovarian tissue in the retroperitoneum. Because of their resemblance to ovarian mucinous cystadenomas, these tumors are thought to arise from heterotopic ovarian tissue, which may explain their occurrence exclusively in women. Furthermore, Subramony et al. [5] reported that the estrogen receptor is positive in stromal cells of a PRMC. An immunohistochemical analysis was found to have a positive match to cytokeratin 7 and cytokeratin 20 antibodies, an identical profile to that of ovarian mucinous tumors [7]. Ovarian remnants, however, have not been identified in the wall of the cysts. In addition, PRMCs have been previously reported as occurring in male patients [2]. The second, more recent hypothesis is that these tumors arise from an invagination of the multipotential mesothelium with subsequent mucinous metaplasia of the mesothelial lining cells, giving rise to a mucinous cyst that enlarges to form a cystic tumor. These multipotential cells undergo metaplasia to the mucinous epithelium, creating mucinous cysts with other malignant phenotypes [4].

Based on a review of the cases reported in the literature, these neoplasms can be classified into three clinicopathologic types. The most common type is the retroperitoneal mucinous cystadenoma, which is a benign cystic tumor characterized by a large, unilocular or multilocular cyst. This tumor behaves in a benign fashion and is not associated with recurrence following resection. In the second type, the lining epithelium contains foci of the proliferative columnar epithelium in addition to the columnar epithelium. This type of tumor resembles an ovarian mucinous tumor of low malignant potential. The third type is the malignant mucinous cystadenocarcinoma. Areas of benign and mucinous tumors of low malignant potential may be seen in addition to the cystadenocarcinoma. These patients may develop a recurrent tumor or die from a metastatic tumor [4].

In rare instances, the diagnosis is often delayed due to the lack of specific presenting symptoms. Mainly, a PRMC presents as a mass either with or without pain. Most patients present with an asymptomatic mass and vague abdominal discomfort. Its clinical presentation depends on the location and the extent of the mass. Preoperative diagnosis is important, as most retroperitoneal masses are malignant. However, this is difficult to achieve because no sensitive methods or reliable markers are available. Moreover, ra- diological imaging, such as CT and MRI, plays an important role in describing and assessing the disease's characteristics and the involvement of adjacent or distant structures of the mass, but it cannot exclude the malignant potential of the retroperitoneal masses. $\mathrm{CT}$ of the area can be beneficial in differentiating between a cystic teratoma and a cystadenoma through detection of calcification within the cyst. Overall, MRI provides an optimal description of the tumor in relation to soft tissue and radiological evidence of the origin of the tumor. On the other hand, serum tumor markers, such as CA 125, CA 19-9, CEA, CA 15-3, and $\alpha$-FP, rarely aid in the diagnosis or follow-up. The aforementioned markers are found to be normal, except in rare cases. Aspiration is a good method to delineate the nature of the cyst, but cytologic analysis of the aspirated fluid frequently fails to reveal the cell type of the epithelial cells of the cyst lining. The CEA level of the aspiration fluid may be measured, and elevation of the CEA level is observed in patients with retroperitoneal mucinous tumors [8]. The final diagnosis remains postoperative and rests on histological examination [9]. A PRMC must be differentiated from other cystic neoplasms, benign or malignant. Benign diseases include lymphoceles, pancreatic pseudocysts, and cystic lymphangiomas. Malignant tumors include cystic teratomas, cystic mesotheliomas, liposarcomas, fibrosarcomas, leiomyosarcomas, malignant fibrous histiocytomas, rhabdomyosarcomas, and pseudomyxoma retro pseudomyxoma retroperitonei peritonei. A PRMC should also be distinguished from tumors of the ovaries or renal tumors [10].

As for the management of PRMCs, complete surgical excision is recommended to eliminate the risk of infection, recurrence, and malignant degeneration. An exploratory laparotomy with complete enucleation of the cyst is traditionally indicated, although successful laparoscopic excision of a PRMC has been reported [4, 8]. However, prevention of cystic fluid spillage during laparoscopic manipulation is important, especially when the pathology of the retroperitoneal cyst is unclear.

In conclusion, when confronted with a cystic mass in the retroperitoneum, a PRMC should be included in the list of differential diagnoses. Complete surgical removal of the tumor is recommended because of the high risk of infection, recurrence, and malignant potential.

\section{CONFLICT OF INTEREST}

No potential conflict of interest relevant to this article was reported.

\section{ACKNOWLEDGMENTS}

This paper was supported by Wonkwang University in 2013.

\section{REFERENCES}

1. Lai EC, Chung KM, Lau WY. Primary retroperitoneal mucinous 
cystadenoma. ANZ J Surg 2006;76:537.

2. Paraskevakou H, Orfanos S, Diamantis T, Konstantinidou A, Patsouris E. Primary retroperitoneal mucinous cystadenoma: a rare case with two cysts and review of the literature. Hippokratia 2014; 18:278-81.

3. Arribas D, Cay A, Latorre A, Cordoba E, Martinez F, Lagos J. Retroperitoneal mucinous cystadenoma. Arch Gynecol Obstet 2004; 270:292-3.

4. Min BW, Kim JM, Um JW, Lee ES, Son GS, Kim SJ, et al. The first case of primary retroperitoneal mucinous cystadenoma in Korea: a case report. Korean J Intern Med 2004;19:282-4.

5. Subramony C, Habibpour S, Hashimoto LA. Retroperitoneal mucinous cystadenoma. Arch Pathol Lab Med 2001;125:691-4.

6. Handfield-Jones RM. Retroperitoneal cysts: their pathology, diag- nosis, and treatment. Br J Sur 1924;12:119-34.

7. Lai KK, Chan YY, Chin AC, Ng WF, Huang YH, Mak YL, et al. Primary retroperitoneal mucinous cystadenoma in a 52-year-old man. J Hong Kong Coll Radiol 2004;7:223-5.

8. Chen JS, Lee WJ, Chang YJ, Wu MZ, Chiu KM. Laparoscopic resection of a primary retroperitoneal mucinous cystadenoma: report of a case. Surg Today 1998;28:343-5.

9. Tangjitgamol S, Manusirivithaya S, Sheanakul C, Leelahakorn S, Thawaramara T, Kaewpila N. Retroperitoneal mucinous cystadenocarcinoma: a case report and review of literature. Int J Gynecol Cancer 2002;12:403-8.

10. Abedalthagafi M, Jackson PG, Ozdemirli M. Primary retroperitoneal mucinous cystadenoma. Saudi Med J 2009;30:146-9. 